\title{
Management of acute myeloid leukaemia
}

\author{
A REGIONAL AUDIT IN THE SOUTH AND WEST OF THE UNITED KINGDOM
}

Simon Rule, Veronique Poirer and Charles Singer

\begin{abstract}
In a retrospective audit data were collected on all 231 patients from 26 hospitals diagnosed with AML in the south and west region of the UK in 1996. Their median age at diagnosis was 67 years. Sixty one percent (142/231) of patients were treated with chemotherapy; most of the rest received blood product support only; and $7 \%$ (15) had no treatment at all. Sixty eight percent of patients aged under 60 years were treated in a clinical trial compared with $24 \%$ of patients over 60 . The major reasons given for not entering patients in a trial were ineligibility in the younger cohort and poor performance status in the older group. Twenty elderly patients (12.5\%) refused to be entered in a trial.

In an unselected cohort of patients with AML the accrual into clinical trials is impressively high for patients under 60 years. However, this is a disease of the elderly and comparable enrolment is not seen with elderly patients despite being managed by the same haematologists. If the percentage of patients entered into trials is to be defined as a quality standard, then it is important for those trials to be relevant to the population being treated.
\end{abstract}

The annual incidence of acute myeloid leukaemia (AML) is approximately 2.4 per $100,000^{1}$, increasing progressively with age to a peak of 12.6 per 100,000 in adults 65 years of age or older. The outcome for patients with AML has improved substantially over the last three decades as a consequence of progress achieved by clinical trials, which have introduced changes in chemotherapeutic drugs and developed dose intensification with bone marrow transplantation. The headline survival figures quoted for such treatments are only applicable to young fit individuals who form the minority of patients, while AML is predominantly a disease of the elderly. There is a clear linear decrease in the percentage of patients achieving a complete remission of leukaemia as age increases, from $70 \%$ in adults under 50 years of age to $22 \%$ in patients over 75 years $^{2}$. Age is an important prognostic factor in virtually all large co-operative group studies ${ }^{3-6}$ due to a number of factors, including an increasing incidence of secondary leukaemias ${ }^{7}$, cytogenetic abnormalities ${ }^{8}$, resistance of leukaemic cells to cytotoxic drugs ${ }^{9}$ and significant co-morbidity.

The standard treatment for these older patients is not clear. Three strategies are commonly employed: standard chemotherapy, attenuated dose chemotherapy or palliative treatment with blood product support only. Although treatment with chemotherapy in this age group achieves better survival than no treatment ${ }^{10,11}$, these trials again involve highly selected patients, and clinical trials in elderly patients have failed to show the improvement in outcome clearly seen in the younger patients.

This audit was undertaken in the south-west of the United Kingdom to examine all patients diagnosed with AML in the calendar year 1996. Our aim was to examine diagnostic and treatment approaches in this unselected population-based cohort with a particular focus on clinical trial participation.

\section{Method}

The south and west Regional Cancer Organisation (RCO) tumour panel in haematological oncology designed a simple single page proforma for data collection. The cohort of patients comprised all newly diagnosed cases of AML presenting during 1996 at each participating centre. All consultant haematologists at 26 acute hospital trusts in the south and west region agreed to take part in the study.

Patient details were collected by the clinical team and completed forms were sent to the RCO office. Data were collected from June 1997 to May 1998 by the central data management team at the RCO office.

\section{Key Points}

$A M L$ is a disease of the elderly

Trial recruitment in the UK for young patients is excellent

Recruitment for the elderly is poor despite being managed by the same physicians, suggesting poor trial design

If patient entry into clinical trials for cancer is to become a quality standard, then those trials must be applicable to the population being treated
Simon Rule FRCPA,

Consultant

Haematologist,

Derriford Hospital, Plymouth

Veronique Poirer Regional Cancer Organisation, Bristol

\section{Charles Singer} FRCP, Consultant Haematologist and Panel Chairman, Bath Royal United Hospital 
Care was taken to exclude patients who may have been entered twice as a result of moving between hospitals within the region during therapy.

\section{Results}

\section{Patient characteristics}

Data were collected on 231 patients. One small trust did not have any cases of AML to report for 1996. The age distribution of the cohort is illustrated in Fig 1. The median age is 67 years and there is a slight male predominance (51\%).

One hundred and thirty three patients (58\%) were diagnosed as primary de novo AML and 87 patients (38\%) were identified as secondary AML; in 11 cases no classification was given. The median age of the primary AML group was 65 years (range 1-89) and the median age of the secondary AML group was 73 years (range 16-93). Most of the patients with secondary leukaemia evolved from a prior myelodysplastic state but eight patients over 60 years $(5 \%)$ and four patients under 60 years (6\%) had an earlier history of exposure to chemotherapy or radiotherapy.

\section{Management}

Chemotherapy was administered to 142 patients (61\%) with a median age of 62 years (range 1-92). Chemotherapy was administered to $71 \%$ of patients with primary de novo AML and $51 \%$ of patients with secondary AML. Eighty eight patients (40\%), median age 78 (range 17-95), received no chemotherapy; no data were available for one patient. Most of these patients received palliative treatment with blood products only; 15 of them (7\%) (median age 78, range 36-95) received no treatment at all either because they refused or died before appropriate therapy could be administered. One young patient who refused all therapy was a Jehovah's Witness.

In 26 patients high dose therapy and autologous or allogeneic bone marrow transplantation was identified as a component of the initial treatment strategy.

\section{Clinical trial involvement}

The involvement of patients in clinical trials by age is shown in Fig 2. No data were available on two patients. Sixty eight percent (47/68) of patients aged less than 60 years were treated in the context of a clinical trial of AML treatment. Of this group of 68 patients (data available on 67 patients) 79\% (42/53) with primary de novo AML entered a clinical trial compared with $36 \%(5 / 14)$ of patients with secondary AML. The main reasons recorded for treatment outside a clinical trial of AML therapy were ineligibility (eight cases), lack of local infrastructure to conduct clinical trials (four cases, one centre), patient refusal to participate (two cases) and poor general fitness (two cases). In six cases no information was provided.

In contrast, only $24 \%$ (38/161) of patients over 60 years received chemotherapy in the context of a clinical trial. This

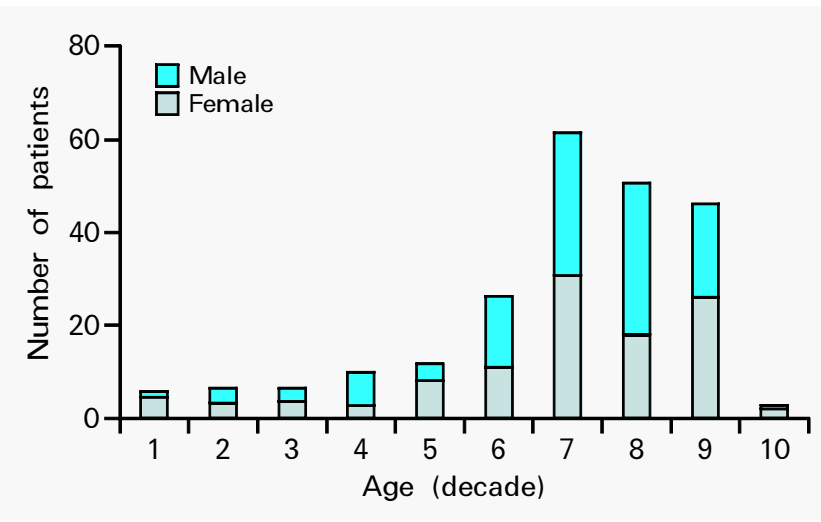

Fig 1. Age and sex distribution of the cohort.

represents $45 \%(38 / 81)$ of patients in this age range who received any form of chemotherapy. The majority of those treated were aged less than 70 and were entered into the Medical Research Council 11 trial for elderly patients with AML. Patients not entered were medically unfit or had a specific contra-indication to treatment, while $12.5 \%(20 / 161)$ refused trial entry.

\section{Outcome}

Complete remission was documented in $54 \%$ of patients aged less than 60 . Of these patients $84 \%$ treated within a clinical trial achieved complete remission. The remission rate was higher in patients with primary de novo AML than in secondary AML (72\% and $17 \%$ respectively). Twenty three patients under 60 are known to have died (34\%), mostly as a consequence of leukaemia. Complete remission was achieved in 32 patients aged over $60(20 \%)$. For patients treated within a clinical trial the complete remission rate is $44 \%$. One hundred and fourteen patients aged 60 years and older are known to have died (69\%), and although the majority died of leukaemia, 31 (27\%) died as a result of complications of treatment.

\section{Discussion}

This is a retrospective study of the incidence, management and outcome of all patients diagnosed with acute leukaemia in the south and west of the United Kingdom over a period of one year. AML is uniformly well diagnosed: in the Medical Research Council AML10 study only 18 of 1966 patients from 163 institutions, throughout the UK and abroad, were excluded owing to mistaken diagnosis ${ }^{12}$. This study represents all patients diagnosed for a population of 6.5 million and demonstrates an annual incidence of 3.5/100,000.

Accepting the limitations of such a study, some clear trends are seen. AML is a disease of the elderly. In this study the median age at presentation was 67 years, which is slightly older than in other studies ${ }^{13,14}$. Many elderly patients have secondary leukaemia arising from an earlier myelodysplastic state but in this unselected cohort only $6 \%$ of patients under 60 and $5 \%$ over 60 had a previous history of exposure to chemotherapy or radio- 
therapy, a rate lower than expected ${ }^{15}$. In the majority of cases and in all hospitals advanced diagnostic techniques are applied. Only in the very elderly are they not used, reflecting the fact that such information is often not relevant to the management of such patients.

Headline treatments such as high dose therapy with autologous or allogeneic bone marrow transplantation ${ }^{16}$, although resource intensive, are only relevant to a minority of patients presenting with this illness.

Information on outcome is limited and no real conclusions can be drawn. It may be inferred that patients entered on trials have a better outcome, but this may simply reflect patient selection.

The accrual of patients under 60 into clinical trials is impressively high. Across a totally unselected population this represents almost $70 \%$ of patients. This demonstrates a genuine commitment from clinicians, as a result of which the outcome for these patients has improved significantly over the last three decades. The outcome for elderly patients with AML has not kept pace with that of younger patients, but it is clear that these patients do better if treated with chemotherapy, prompting suggestions that all elderly patients should receive full dose chemotherapy ${ }^{11}$. This advice is based on published studies of highly selected patients and in some cases emanates from referral centres that fail to see the true spectrum of patients reported here. There is clearly a subset of elderly patients who are amenable to treatment; they have received therapy and some have been entered into studies. Why has the majority of older patients not been enrolled in trials? For trials to recruit successfully, two factors are needed. First, a commitment on the part of the clinician to the process which includes having the necessary infrastructure; second, studies that are applicable and acceptable to both doctor and patient. The same haematologists who enthusiastically enrol young patients into studies fail to enter the elderly. This suggests that trials in this age group must be reexamined. Perhaps trials for this group of patients should aim primarily to examine quality of life issues rather than to attain complete remission with increasingly complicated and intensive chemotherapy.

\section{Fig 2. Patient entry into clinical trial.}

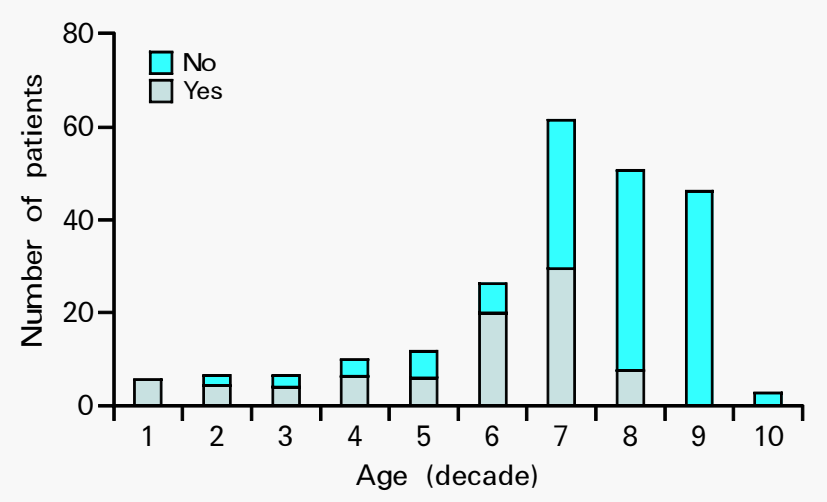

This has broader implications. At a time when we are starting to define acceptable standards of care for cancer it is important that these are based not on published - and therefore, by definition, selected - patients but on population based standards. In a population based study from Toronto overall the complete remission rate of all patients diagnosed with AML was $43.8 \%{ }^{17}$. However, when the usual exclusions were applied this rose to $85.3 \%$. It is clear from this study and our own that the results from clinical trials only apply to a minority of patients, yet if the percentage of patients entered into trials is to be defined as a quality standard, then it is important for those trials to be relevant to the population that is being treated.

\section{Members of panel}

Charles Singer (Chair), Brian Attock, Andrew Bell, Sally Chown, Roger Evely, Mike Hamon, Chris James, Steve Kelly, Han Mynt, Hugh Parry, Marilyn Pocock, Chris Price, Nick Rooney, Janet Ropner, Simon Rule, Mark Smith and John Sweetenham.

\section{References}

1 Kosary CL, Ries LAG, Miller BA, Hankey BF, Edwards BK (Eds). SEER Cancer Statistics Review, 1973-1992: tables and graphs. Bethesda, Maryland: National Cancer Institute, 1995.

2 Johnson PRE, Liu Yin JA. Acute myeloid leukaemia in the elderly: biology and treatment. Br J Haematol 1993; 83:1-6.

3 Glucksberg H, Cheever MA, Farewell VT, Fefer A, et al. High dose combination chemotherapy for acute non-lymphoblastic leukaemia in adults. Cancer 1981;48:1273-81.

4 Rai KR, Holland JR, Glidewell OJ, Weinberg V, et al. Treatment of acute myelocytic leukaemia: a study by Cancer and Leukaemia Group B. Blood 1981; 58:1203-12.

5 Rees JK, Gray RG, Swirsky D, Hayhoe FG. Principal results of the Medical Research Council's 8th acute myeloid leukaemia trial. Lancet 1986;2:1236-41.

6 Rees JK, Gray RG, Wheatley K. Dose intensification in acute myeloid leukaemia: greater effectiveness at lower cost - principal report of the Medical Research Council's AML9 study. Br J Haematol 1996;94:89-98.

7 Hoyle CF, de Bastos M, Wheatley K, Sherrington PD, et al. AML associated with previous cytotoxic therapy, MDS or myeloproliferative disorder: results from the MRC's 9th AML trial. Br J Haematol 1989; 72:45-53.

8 Swansburg GJ, Lawler SD, Alimena G, Arthur D, et al. Long-term survival in acute myelogenous leukaemia: a second follow-up of the Fourth International Workshop on Chromosomes in Leukaemia. Cancer Genet Cytogenet 1994; 73:1-7.

9 Wood P, Burgess R, MacGregor A, Lin Yin JA. P-glycoprotein expression on acute myeloid leukaemia blast cells at diagnosis predicts response to chemotherapy and survival. Br J Haematol 1994; 87:509-14.

10 Lowenberg B, Zittoun R, Kerkhofs $\mathrm{H}$, Jehn U, et al. On the value of intensive remission-induction chemotherapy in elderly patients of $65+$ years with acute myeloid leukaemia: a randomised phase III study of the European Organisation for Research and Treatment of Cancer Leukaemia Group. J Clin Oncol 1989;7:1268-74.

11 Hiddemann W, Kern W, Schoch C, Fonatsch C, et al. Management of acute myeloid leukaemia in elderly patients. J Clin Oncol 1999;17:3569-76.

12 Burnett AK, Goldstone AH, Stevens RMF, Hann IM, et al. Randomised comparison of addition of autologous bone-marrow transplantation to intensive chemotherapy for acute myeloid leukaemia in first remission: results of MRC AML 10 trial. Lancet 1998; 351:700-8. 
13 Surveillance and mortality data, 1973 to 1977. US Department of Health and Human Services.

14 Harousseau J-L. Acute myeloid leukaemia in the elderly. Blood Rev 1998;12:145-53.

15 Pedersen-Bjergaard J, Peredson M, Roulston D, Philip P. Different genetic pathways in leukaemogenesis for patients presenting with therapy related myelodysplasia and therapy related acute myeloid leukaemia. Blood 1995;86:3542-52.

16 Cassileth PA, Harrington DP, Appelbaum FR, Lazarus HM, et al. Chemotherapy compared with autologous or allogeneic bone marrow transplantation in the management of acute myeloid leukaemia in first remission. N Engl J Med 1998;339:1649-56.
17 The Toronto Leukaemia Study Group. Results of chemotherapy for unselected patients with acute myeloblastic leukaemia: effect of exclusions on interpretation of results. Lancet 1986; 1:786-8.

Address for correspondence: Dr Simon Rule Consultant Haematologist, Department of Haematology, Derriford Hospital, Plymouth, Devon PL6 8DH

E-mail: simon.rule@phnt.swest.nhs.uk

\section{Acute Myocardial Infarction A core data set for monitoring standards of care}

\section{Prepared on behalf of the National Service Framework for Coronary Heart Disease}

The core data set for monitoring the standards of care for myocardial infarction is the product of a broad collaboration initiated by the Clinical Effectiveness and Evaluation Unit (CEEu) of the Royal College of Physicians and involving cardiologists, representatives of management, nursing, the ambulance service and the Department of Health. The definitions used in the data set are as specified in the recently announced National Service Framework (NSF) for Coronary Heart Disease, which demands high standards for the care of patients with myocardial infarction.

The data set will allow the nationwide examination of care, help clinicians to examine the quality of care they offer and permit valid comparisons to be made between hospitals. It will also encourage clinicians to be involved in the audit process from the outset and provides a tool for them to ensure that data is accurate and complete. There is evidence that the care of patients following myocardial infarction, in particular the use of treatments aimed at reducing the risk of further events, can be improved. This work will allow the use of treatments to be monitored countrywide.

The CEEu will be working with the National Institute for Clinical Effectiveness (NICE) to collect data from across the country to make sure that the results are meaningful, credible and useful.

CONTENTS: Introduction I Acute myocardial infarction: a clinical overview I Principles underlying the core data set I How should the data be acquired and from whom? Data processing analysis and security Data interpretation I How should the data be used?

CORE DATA SET: 1. Demography; 2. Delays in treatment; 3. Cardiac arrest and resuscitation; 4. Thrombolytic and anti-thrombotic therapy; 5. Hospital course; 6. Secondary prevention initiated in hospital; 7. Investigations performed or recommended.

APPENDIX: Core data set fields according to the originating source and specification for each field, grouped into three categories.

\section{AVAILABLE FROM THE ROYAL COLLEGE OF PHYSICIANS}

ISBN 1860161197 A4 report, soft cover, 34 pages

Price: UK $£ 12.00$, overseas $£ 15.00$ (prices include postage and packing) 